\author{
Konstantinos Papadopoulos* and \\ Russell Kabir \\ Anglia Ruskin University, Allied and Public Health \\ Department, Faculty of Medical Sciences, UK \\ Dates: Received: 27 October, 2016; Accepted: 05 \\ November, 2016; Published: 07 November, 2016 \\ *Corresponding author: Dr Konstantinos \\ Papadopoulos, Anglia Ruskin University, Allied and \\ Public Health Department, Faculty of Medical Sciences, \\ Chelmsford, CM1 1SQ, UK, Tel: +44 (0) 01245 68442; \\ E-mail: Kostas.Papadopoulos@anglia.ac.uk \\ www.peertechz.com \\ ISSN: 2455-5487
}

Keywords: Patellofemoral Pain Syndrome; Pain; Function; Strengthening; Stretching; Physiotherapy effectiveness

\section{Research Article \\ Physiotherapy Effectiveness on Muscle Strength, Flexibility, Pain and Function in Patients with Patellofemoral Pain Syndrome}

\section{Introduction}

Patellofemoral Pain Syndrome (PFPS) is one of the most common knee conditions that physiotherapists and physicians have to confront Houghton, 2007. PFPS often becomes chronic, with up to $91 \%$ of patients reporting constant knee pain 4-18 years after being diagnosed [1], whilst 5 years after rehabilitation, $80 \%$ still reported pain and $74 \%$ had reduced their activity level [2]. A search of the literature did not reveal previous research studies which have investigated the effectiveness of the National Health Service (NHS) physiotherapy treatment of PFPS in a non- academic setting. The last update of the physiotherapy standards of proficiency [3], reported that registered physiotherapist should use specific practice skills such as exercise and movement to enhance functional ability. Literature showed a number of different treatment components that clinical physiotherapists are suggested to use when treating patients with PFPS and all of them included strengthening and stretching of several muscles [4-6]. Previous research identified the clinical methods that North-West Wales (NWW) physiotherapists use when treating patients with PFPS [7]. In that study NWW physiotherapists reported using a selection of physical activities aimed at strengthening and stretching lower limb muscles. However, there was no evidence about the effectiveness of such treatment in NHS physiotherapy departments. Harvie et al. [6], reported in their review that a sixweek intervention should be considered for programmes targeting patients with PFPS. In addition, literature [8] and bibliography [9], suggest that six weeks can be enough if intense and specific strength and flexibility programs. This study not only will reveal the effect of a six-week physiotherapy treatment in district NWW NHS hospitals, but will also provide answers to the efficacy of this exercise-based treatment to the PFPS population who visit district physiotherapy clinics.

\section{Aims}

The aim of this study was:

- To investigate the effect on muscle strength, flexibility, pain and function of a six week programme of physiotherapy for PFPS delivered in a district NWW NHS hospital.

\section{Methods}

Recruitment method: All potential participants were identified by an extended scope physiotherapist who searched the NHS physiotherapy referrals (MB). An invitation letter and an information sheet were then sent to them. Potential participants were phoned by the rheumatologist (JJ) and if they were interested the phone was passed to the researcher who arranged a meeting after the first physiotherapy appointment. Participants were given at least two days to decide whether to participate. The first assessment took place directly after the first treatment session with the physiotherapist; the 
second assessment took place directly the last physiotherapy visit which was approximately after 5-8 weeks.

Ethical approval was granted by the local NHS research ethics committee (10/WNo01/60). Informed consent was obtained from all participants before data collection was initiated.

\section{Participant inclusion/exclusion criteria}

The inclusion criteria for all patients were to be:

- Referred by a general practitioner or a consultant for assessment and treatment of PFPS

- Aged between 18 to 50 years old

- Able to communicate in English.

- Able to participate in both sessions (First and last visit time at the physiotherapy department)

The exclusion criteria for the PFPS group included the presence of other knee conditions such as knee ligament conditions/menisci conditions, history of trauma, previous knee surgery, history of true locking, history of patellar dislocation, history of arthritis, knee joint effusion, patellar tendinopathy, or the inability to attend all sessions [10].

\section{Procedure}

After participants had received their first session of physiotherapy they met the researcher who was blinded to the patients' diagnoses and asked them to complete a consent form. Details of the participants' weight, height and age were recorded. Participants were then asked to complete a series of questionnaires and scales. Following this, a number of physical tests were performed.

After they had received their final treatment and were ready for discharge, participants filled in the questionnaire and scales again and repeated the clinical tests. In addition, after the follow-up session all participants received $£ 10$ in high street vouchers. Participants completed a form confirming they had received the vouchers.

\section{Questionnaires and scales}

The Anterior Knee Pain Scale (AKPS) [11], is a very common scale in the assessment of PFPS [12]. This scales has been designed to measure dysfunction, disability and pain whilst in the past, it has been used in several PFPS studies showing high reliability $(r>0.9)[13,14]$.

Along with the AKPS, two more VASs for usual pain and for pain on the day of the assessment were also used. Participants were asked to pencil vertically on a $10-\mathrm{cm}$ line (from 'no pain' to 'pain as bad as it could possibly be') according to the usual pain and the pain they had on the day of the assessment. The questionnaire and the VASs were completed at both sessions.

\section{Flexibility tests}

Two tests were performed:

- The Modified Thomas test measuring hip and knee flexion.

This test is designed to report tightness of iliopsoas and quadriceps. To measure iliopsoas tightness, the axis of the goniometer (Absolute Axis, Baseline, New York, USA) was positioned on top of the greater trochanter, with one arm placed parallel to the longitudinal axis of femur and the other arm parallel to the mid-axillary line of the trunk. To measure quadriceps tightness, the axis of the goniometer was placed on the head of the fibula on the examined leg, with one arm being parallel to the longitudinal axis of the tibia pointing toward the lateral malleolus and the other parallel to the longitudinal axis of femur pointing towards the greater trochanter. The non-examined leg was fully flexed and held by the participant's hands while the tested leg was extended by gravity. The maximal hip extension and knee flexion range of motion values were recorded when the gravity could not further extend the hip and flex the knee. No additional passive movement was performed by the researcher's hands in either hip extension or knee flexion [15]. A more positive hip angle value represents more severe tightness of iliopsoas whereas a more positive knee extension angle represents more severe tightness of quadriceps.

- The hamstrings flexibility test.

This test measures the flexibility of the hamstrings. Participants were in supine position. Two straps were placed, one on the non-examined leg; across the thigh and a second over the anterior superior spines of the ilia to stabilize the pelvis. A line was drawn between the fibular head and lateral malleolus of the leg. This line represented the longitudinal axis of the leg and was a reference of accurate placement of the goniometer. The examiner placed the hip to 90 degrees (confirmed by a goniometer) whilst participants held their knee flexed and the foot in plantar flexion. Then, with the hip stabilized at 90 degrees, participants actively extended the knee until they reached initial mild resistance. The angle of the knee was then recorded by the goniometer as the flexibility of the hamstring muscles [16].

\section{Strength tests}

Participants were then asked to complete a series of strength tests on a portable dynamometer which has been found to be reliable and valid [17]. Seven contractions (each of them lasting for five seconds) were completed for each test. The first three were a warm up of $25 \%, 50 \%$ and $75 \%$ of their maximum strength followed by four maximal voluntary contractions (MVCs). Only the strongest MVC was recorded. There was a 30 seconds rest between contractions and a two-minute rest between tests. The researcher encouraged the participants verbally.

The tests involved:

- Isometric knee extensions from sitting position with the knee extended to $60^{\circ}$.

This test measures the strength of the quadriceps muscles. Participants were placed in a sitting position with the knee extended at 60 degrees of full length extension and asked to forcibly extend their knee against the dynamometer. The hip was flexed at 90 degrees whilst the trunk, pelvis and foot were strapped tight with belts [18]. 
- Isometric hip external rotation from supine position with the tested leg rotated externally to 5 degrees.

In supine position with both knees fully extended and the tested leg externally rotated to 5 degrees, participants were asked to rotate the foot externally against the resistance of the dynamometer. The pelvis and the tested knee were strapped with belts as no pelvic movement or knee flexion were allowed [19].

- Isometric hip abduction from 'clam' position.

The set up for the 'clam' test was the same as the isometric hip abduction from 'clam' test position. All participants were asked to perform 2 sets of 30 concentric hip abduction repetitions (60 degrees/sec) against the resistance of the isometric dynamometer. The range of motion was from 0 to 30 degrees. The mean score of the 30 repetitions was calculated ( $\mathrm{N} \mathrm{x} \mathrm{m/weight)} \mathrm{[20].}$

\section{Functional stress protocol}

Participants then performed a functional stress protocol involving two sets of 30 repetitions from a 'clam' position to 30 degrees of hip abduction with a red thera-band" (The Hygenic corporation, Akron Ohio, USA) around both of their knees. The red thera-band (4 pounds force in $100 \%$ elongation) is the elastic band the physiotherapists use in the clinic when they prescribe resistance exercises to their participants. The band was tied tightly around the knees. During the protocol the researcher held a t-shape standing measure which was adjusted to show each participant the position of 30 degrees of hip abduction. The knee had to touch the t-shape tool on each repetition of the protocol. There was a two-minute break between the two sets.

As soon as the functional stress protocol was completed two more maximum isometric contractions from 'clam' test position were immediately performed using the portable dynamometer. The first contractions was used to identify how much the functional stress protocol had reduced the participant's activity and the second, which took place two minutes later, was used to assess the rate of recovery.

\section{Data and statistical analysis}

A goniometer (Absolute Axis, Baseline, New York, USA) was used to measure all flexibility tests in degrees, while for the strength tests a peak value was measured using chart $5^{\circ}$ software for windows. Only the highest out of the 4 MVCs was used for analysis. The strength produced was normalized by participant weight.

Paired t-tests were used to identify the effect of physiotherapy treatment in patients with PFPS after a six-week physiotherapy treatment.

The effect size ' $r$ ' was also measured by using the Cohen's d given by the equation:

$$
d=\frac{\chi \text { pre }-\chi \text { post }}{S D}
$$

where $\chi$ pre was the average mean of pre-treatment, $\chi$ post was the average mean of post-treatment and SD was the Standard Deviation [21]. When using this equation the effect sizes ' $r$ ' of .20 are small, .50 are medium, and .80 are large. Chi-square tests for the diagnostic tests before and after treatment were also performed whilst paired t-tests were performed to identify difference between pre and post 'clam' activity; before and after treatment.

\section{Ethical approval}

The study was approved by the ethics committee of the School of Sport Health and Exercise Sciences and the Betsi Cadwaladr University Health Board (10/WNo01/60).

\section{Results}

\section{Participants}

26 patients with PFPS were referred by a consultant or general practitioner to NWW physiotherapy department were recruited after the recruitment procedure explained in the methods. Table 1, shows the characteristics of the PFPS patients. Only 4 PFPS patients reported permanent pain, while the rest (22) reported on/off pain.

\section{Questionnaire and scales}

The AKPS showed significant improvement in PFPS patients after they received their treatment. The two VAS (the VAS for the pain on the day of the assessment and the VAS for usual pain) showed significant change after treatment.

\section{Flexibility tests}

The analysis of the flexibility tests showed that there was no significant effect $(\mathrm{p}<0.05)$ of the six-week treatment on the length of the quadriceps and iliopsoas muscles. Additionally, the effect sizes ' $r$ ' were small for all tests (Table 2).

\section{Strength tests}

The series of strength tests showed that there was no difference after treatment (Table 2). Figure 1 displays the strength results. Strength tests similar to flexibility tests reported small effect sizes ' $r$ '.

\section{‘Clam' functional stress protocol}

The 'clam' functional stress protocol showed that although strength was reduced before and after the 'clam' test protocol at both time points (Table 2), the physiotherapy treatment had no effect in this performance decrement (Pre functional stress 'clam', $\mathrm{p}=0.36$; Post 1 functional stress 'clam', $\mathrm{p}=0.13$; Post 2 functional stress 'clam', $\mathrm{p}=0.26$ ). All these tests reported moderate effect sizes ' $r$ '.

\section{Discussion}

The main finding of this study was that the patients with PFPS

Table 1: Subject characteristics of the PFPS and non-PFPS groups.

\begin{tabular}{|l|l|}
\hline Age (years) & $35.0 \pm 9.1$ \\
\hline Height (meters) & $1.72 \pm 0.09$ \\
\hline Weight (kilograms) & $77.4 \pm 18.85$ \\
\hline Gender (male/female) & 9 males/17 females \\
\hline $\begin{array}{l}\text { Type of pain } \\
\text { Active patients }\end{array}$ & $\begin{array}{l}4 \text { permanent / } 22 \text { on/off } \\
12 \text { athletes/14 non-active }\end{array}$ \\
\hline
\end{tabular}

The values (except for gender permanent pain and active patients) are presented as mean \pm SD. 
Table 2: The table presents the effect of treatment on flexibility, strength tests, 'clam' stress protocol and questionnaires and scales.

\begin{tabular}{|c|c|c|c|c|}
\hline Flexibility tests & $\begin{array}{l}\text { Before } \\
\text { treatment }\end{array}$ & $\begin{array}{c}\text { After } \\
\text { treatment }\end{array}$ & $\begin{array}{l}p \text { values } \\
\text { following } \\
\text { treatment }\end{array}$ & Effect sizer \\
\hline $\begin{array}{l}\text { iliopsoas flexibility } \\
\text { (degrees) }\end{array}$ & $5.84 \pm 6.89$ & $4.24 \pm 5.59$ & 0.13 & 0.13 \\
\hline $\begin{array}{l}\text { quadriceps flexibility } \\
\text { (degrees) }\end{array}$ & $62.72 \pm 14.31$ & $64.16 \pm 14.19$ & 0.33 & -0.05 \\
\hline $\begin{array}{l}\text { hamstrings flexibility } \\
\text { (degrees) }\end{array}$ & $33.48 \pm 17.98$ & $30.84 \pm 15.48$ & 0.49 & 0.07 \\
\hline \multicolumn{5}{|l|}{ Strength tests } \\
\hline $\begin{array}{l}\text { isometric knee } \\
\text { extension } \mathrm{N} / \mathrm{kg}\end{array}$ & $4.99 \pm 1.71$ & $5.13 \pm 1.41$ & 0.39 & -0.04 \\
\hline $\begin{array}{l}\text { isometric hip } \\
\text { abduction from 'clam' } \\
\text { position N/kg }\end{array}$ & $2.22 \pm 0.76$ & $2.39 \pm 0.76$ & 0.14 & -0.11 \\
\hline $\begin{array}{l}\text { isometric hip } \\
\text { external rotation } \mathrm{N} / \mathrm{kg}\end{array}$ & $1.13 \pm 0.57$ & $1.15 \pm 0.37$ & 0.80 & -0.02 \\
\hline \multicolumn{5}{|l|}{$\begin{array}{l}\text { ‘Clam' stress } \\
\text { protocol }\end{array}$} \\
\hline $\begin{array}{l}\text { Pre functional stress } \\
\text { 'clam' activity N/kg }\end{array}$ & $1.79 \pm 0.79$ & $2.12 \pm 0.80$ & 0.17 & -0.20 \\
\hline $\begin{array}{l}\text { Post } 1 \text { functional } \\
\text { stress 'clam' activity } \\
\text { N/kg }\end{array}$ & $1.46 \pm 0.65$ & $1.67 \pm 0.73$ & 0.26 & -0.15 \\
\hline $\begin{array}{l}\text { Post } 2 \text { functional } \\
\text { stress 'clam' activity } \\
\mathrm{N} / \mathrm{kg}\end{array}$ & $1.52 \pm 0.61$ & $1.72 \pm 0.72$ & 0.26 & -0.14 \\
\hline \multicolumn{5}{|l|}{$\begin{array}{l}\text { Questionnaires and } \\
\text { Scales }\end{array}$} \\
\hline AKPS & $66.23 \pm 16.26$ & $72.04 \pm 12.83$ & $0.01^{*}$ & -0.19 \\
\hline VAS usual pain & $4.32 \pm 2.74$ & $3.29 \pm 2.33$ & $0.02^{*}$ & 0.20 \\
\hline VAS on the day & $2.35 \pm 2.33$ & $1.59 \pm 1.87$ & $0.02^{*}$ & 0.18 \\
\hline
\end{tabular}

\section{Strength tests}

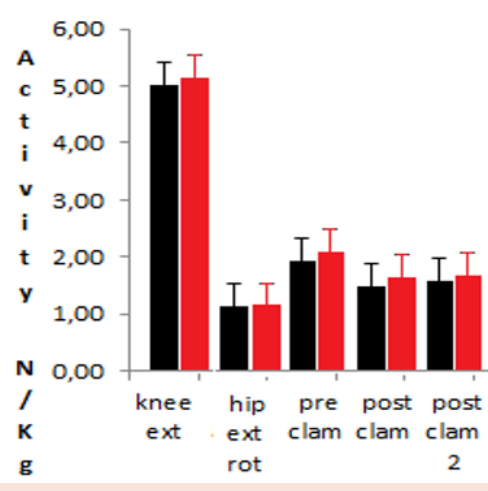

Figure 1: The figure displays the isometric strength tests prior to and following physiotherapy treatment.

improved regarding their pain and function following physiotherapy treatment at district NHS hospitals, while their strength and flexibility did not. The AKPS showed that patients with PFPS got discharged with significant better function. The two VAS (for usual pain and for pain the day of the assessment) reported significant change after treatment; the VAS for usual pain could detect the average pain patients were experiencing, but as PFPS reports more an on/off pain than a constant pain, a VAS for the pain on the day of the assessment was deemed necessary.

\section{Treatment effect}

Although the pain was shown to be reduced after treatment, there was not a significant increase in strength or flexibility post treatment. In addition to reducing pain and improving function physiotherapists may well be aiming to increase strength and flexibility as they reported in previous research study conducted in the same NHS physiotherapy departments [7]. However this study showed that this treatment components were not achieved. It seems that although physiotherapists probably aimed to increase strength and flexibility, they only improved function and pain thus the AKPS and the two VASs improved. Cook et al. [22], suggested the pain during a single squat as another outcome measure to detect physiotherapy effect. In the current study the 'clam' performance was used. However, physiotherapy treatment had no effect on the 'clam' performance decrement. The non-significant results in strength and flexibility are not in line with the systematic review of Harvie et al. [6], who reported that a six-week exercise intervention could be effective (strength and flexibility-wise) for patients with PFPS. Same perception is reported by (Kisner and Colby, 2012). However, the authors reported that the majority of studies, prescribed five or more days of exercises per week and the intensity of the exercises was high. A similar review from Bolgla and Malone [23], included studies with a minimum of 12 visits in a four week intervention. This frequency of visits is not possible in a NHS physiotherapy clinic where physiotherapists see their patients once per week at maximum whilst the session per patients is 20 minutes only. Additionally, if the exercises were prescribed for home use, neither the compliance nor the intensity could be guaranteed since physiotherapists reported [7], that their patients often do not comply with their exercises. Noncompliance may be related to the fact that many patients are not used to exercising and in this study most of the patients were not athletes (14/26) and did not previously perform any sports exercises or sport activities. The decrease in pain following treatment may have thus come from the factors, other than strength improvements, reported in previous PFPS reviews such as patient education - including activity recommendations, sham treatments, low intensity exercises and antiinflammatory or analgesic drugs [5]. Frye et al. [5], report that these modalities have been found to play an important role in improving patient outcomes. However, because of the plethora of interventions they state it is difficult to isolate the precise source of improvement.

\section{Limitations}

The sample size was calculated according to the needs of a comparison study (to report differences between PFPS and other conditions group) and not to the needs of an intervention study. In addition, the frequency that patients visited the physiotherapy department was not recorded for this study. However, we know that all patients visited the clinic at least once per week. In addition, the patients were not asked whether or how often they used pain medication. 


\section{Conclusions/Implications}

Physiotherapy treatment did not improve strength or flexibility in this feasibility study. Treatment did however, improve pain and function. There are several possible explanations for this including the therapeutic effect of the physiotherapeutic consultation, explanation of the cause of the knee condition and what the patient could do about it, a placebo effect and improving posture and the quality of movement. Future studies should identify the possible barriers in the fully adoption and implementation of the suggested research interventions by the NWW physiotherapists. If their major aim was to improve pain and function, then their treatment works. If this turns out to be the case then there is a need to look at the implications of this practice.

\section{Acknowledgement}

The authors would like to thank Moyra Barnes and Dr Jeremy Jones for helping in the recruiting process.

\section{Funding}

The study was funded by the Research Collaboration Building Capacity (RCBC), Wales.

\section{References}

1. Stathopulu E, Baildam E (2003) Anterior Knee Pain: a long term follow-up. Rheumatology 42: 380-382.

2. Noehren B, Scholz J, Davis I (2011) The effect of real-time gait retraining on hip kinematics, pain and function in subjects with patellofemoral pain syndrome. Br J Sports Med 45: 691-696.

3. Health \& Care Professions Council (2013) Standards of proficiencyphysiotherapists, Publication code: 20120301jPOPUB.

4. Lankhorst NE, Bierma-Zeinstra SM, van Middelkoop M (2012) Risk factors for patellofemoral pain syndrome: A systematic review, Journal of Orthopaedic \& Sports Physical Therapy 42: 81-94.

5. Frye JL, Ramey LN, Hart JM (2012) The effects of exercise on decreasing pain and increasing function in patients with patellofemoral pain syndrome: $A$ systematic review. Sports Health 4: 205-210.

6. Harvie D, O'leary T, Kumar S (2011) A systematic review of randomized controlled trials on exercise parameters in the treatment of patellofemoral pain: what works? J Multidiscip Healthc 4: 383-392.

7. Papadopoulos K, Noyes J, Barnes M, Jones GJ, Thom MJ (2012) How do physiotherapists assess and treat patellofemoral pain syndrome in North Wales? A mixed method study. Int J Ther Rehab19: 261-272.
8. Micheo W, Baerga L, Miranda G (2012) Basic principles regarding strength, flexibility and stability exercises. PM R: the journal of injury, function and rehabilitation 4: 805-811.

9. Kisner C, Colby AL (2012) Therapeutic exercise: foundations and techniques, sixth edition. Philadelphia: F.A. Davis Company.

10. Crossley K, Bennell KL, Green S, Cowan S, McConnell J (2002) Physical therapy for patellofemoral pain: a randomized, double blinded, placebocontrolled trial. Am J Sports Med 30: 857-865.

11. Kujala UM, Jaakkola LH, Koskinen SK, Taimela S, Hurme M, et al. (1993) Scoring for Patellofemoral Disorders. Arthroscopy 9: 159-163.

12. Howe TE, Dawson LJ, Syme G, Duncan L, Reid J (2012) Evaluation of outcome measures for use in clinical practice for adults with musculoskeletal conditions of the knee: A systematic review. Man Ther 17: 100-118.

13. Crossley KM, Bennell KL, Cowan SM, Green S (2004) Analysis of outcome measures for persons with patellofemoral pain: which are reliable and valid? Arch Phys Med Rehabil 85: 815-822.

14. Bennell K, Bartam S, Crossley K, Green S (2000) Outcome measures in patellofemoral pain syndrome: test retest reliability and inter-relationships. Physical Therapy in Sport 1: 22-41.

15. Harvey D (1998) Assessment of the flexibility of elite athletes using the modified Thomas test. Br J Sports Med 22: 68-70.

16. Gajdosik R, Lusin G (1983) Hamstring muscle tightness: Reliability of an active knee-extension test. Physical Therapy 63: 1085-1088.

17. Papadopoulos K, Stasinopoulos D (2016) Reproducibility of lower strength tests using a portable dynamometer. Measurement comparisons with a nonportable dynamometer. Int J Phy Ther Rehab 2:1-7.

18. Welsch MA, Williams PA, Pollock ML, Graves JE, Foster DN, et al. (1998) Quantification of full-range-of-motion unilateral and bilateral knee flexion and extension torque ratios. Arch Phys Med Rehabil 79: 971-978.

19. Willson JD1, Binder-Macleod S, Davis IS (2008) Lower extremity jumping mechanics of female athletes with and without patellofemoral pain before and after exertion. Am J Sports Med 36: 1587-1596.

20. Distefano LJ1, Blackburn JT, Marshall SW, Padua DA (2009) Gluteal Muscle Activation during Common Therapeutic Exercises. J Orthop Sports Phys Ther 39: 532-540.

21. Thalheimer W, Cook $S$ (2002) How to calculate effect sizes from published research: A simplified methodology, A Work-Learning Research Publication.

22. Cook C, Hegedus EJ, Hawkins R, Scovell F, Wyland D (2010) Diagnostic accuracy and association to disability of clinical test findings associated with patellofemoral pain syndrome. Physiotherapy Canada 62: 17-24.

23. Bolgla LA, Malone TR (2005) Exercise prescription and patellofemoral pain: evidence for rehabilitation. J Sports Rehab 14: 72-88.

Copyright: (C) 2016 Papadopoulos K, at al. This is an open-access article distributed under the terms of the Creative Commons Attribution License, which permits unrestricted use, distribution, and reproduction in any medium, provided the original author and source are credited. 